\section{The effect of airway pressure and oscillation amplitude on ventilation in pre-term infants}

\author{
Martijn Miedema*, Frans H. de Jongh*, Inez Frerichs", Mariette B. van Veenendaal* \\ and Anton H. van Kaam*
}

ABSTRACT: We determined the effect of lung recruitment and oscillation amplitude on regional oscillation volume and functional residual capacity (FRC) in high-frequency oscillatory ventilation (HFOV) used in pre-term infants with respiratory distress syndrome (RDS).

Changes in lung volume, oscillation volume and carbon dioxide levels were recorded in $\mathbf{1 0}$ infants during a stepwise recruitment procedure, and an increase in pressure amplitude of $5 \mathrm{cmH}_{2} \mathrm{O}$ was measured using electrical impedance tomography and transcutaneous monitoring. The pressures at maximal respiratory system compliance, maximal oscillation volume and minimal carbon dioxide levels were determined. Impedance data were analysed for the chest cross-section and predefined regions of interest.

Despite the fixed pressure amplitude, the oscillation volume changed during the incremental pressure steps following a parabolic pattern, with an inverse relationship to the carbon dioxide pressures. The pressures corresponding with maximal compliance, maximal oscillation volume and minimal carbon dioxide were similar and highly correlated. Regional analysis showed similar findings. The increase in pressure amplitude resulted in increased oscillation volumes and decreased carbon dioxide levels, while FRC remained unchanged.

In HFV pre-term infants with RDS, oscillation volumes are closely related to the position of ventilation in the pressure-volume envelope and the applied pressure amplitude. Changes in pressure amplitude do not seem to affect FRC.

KEYWORDS: Electrical impedance tomography, high-frequency oscillatory ventilation, oscillation volume, pre-term infant

$\mathbf{H}$ igh-frequency oscillatory ventilation (HFOV) is a lung protective ventilation mode frequently used in pre-term infants with respiratory failure [1]. It has been suggested that ventilation and oxygenation during HFOV can be controlled independently by adjusting, respectively, the oscillation amplitude or frequency, and the continuous distending pressure (CDP) or the fraction of inspired oxygen $\left(\mathrm{FI}_{1} \mathrm{O}_{2}\right)$ [2-6]. An increase in oscillation amplitude at a fixed frequency will increase the oscillatory volume measured at the airway opening and improve carbon dioxide removal [7-9]. An increase in CDP at a fixed $\mathrm{F}, \mathrm{O}_{2}$ will increase lung volume by alveolar recruitment in atelectatic lung diseases and improve oxygenation by reducing the intrapulmonary right-to-left shunt $[5,10]$.

From a physiological standpoint it is unlikely that the control of ventilation and oxygenation are truly independent from each other during HFOV [11-13]. Changes in functional residual capacity (FRC) during a recruitment procedure will almost certainly impact on lung compliance and oscillation volume ( $V$ osc), as the position of ventilation changes along the inflation and deflation limbs of the pressure-volume curve [12-15]. Furthermore, adjustments in oscillation amplitude also may, in theory, lead to (small) changes in alveolar pressures, which in turn can affect FRC.

To date, there are no studies exploring the effect of FRC on ventilation and vice versa in highfrequency ventilated pre-term infants with respiratory distress syndrome (RDS). An important reason for this knowledge gap is the lack of an easy to use bedside tool for continuous monitoring of lung volume in pre-term infants. This has recently changed with the introduction of electrical impedance tomography (EIT). EIT can continuously

\section{AFFILIATIONS}

*Dept of Neonatology, Emma Children's Hospital, Academic Medical Center, Amsterdam, The Netherlands.

\#Dept of Anesthesiology and Intensive Care Medicine, University Medical Center Schleswig-Holstein, Campus Kiel, Kiel, Germany.

CORRESPONDENCE

M. Miedema

Dept of Neonatology (H3-214) Emma Children's Hospital AMC P0 Box 22660 1100 DD Amsterdam the Netherlands E-mail: m.miedema@amc.uva.nl

Received: Aug 122011 Accepted after revision: Jan 082012 First published online: Feb 232012 
and noninvasively monitor changes in lung impedance, which are highly correlated with changes in gas volume [16]. EIT provides regional information on lung volume changes in the chest crosssection, which may be important in case of heterogeneous lung disease or gravitational effects [16-19].

In our study, we used EIT to assess the impact of FRC changes during an individualised lung recruitment procedure in preterm infants with RDS on $V$ osc. In addition, we studied the effect of a standardised increase in oscillation amplitude on $V$ osc and FRC. We hypothesised that $V_{\text {osc }}$ changes in relation to the position of ventilation on the pressure-volume curve and that changes in oscillation amplitude will impact on $V$ osc but not FRC.

\section{METHODS \\ Patients}

The study was performed in the neonatal intensive care unit of the Emma Children's Hospital, Academic Medical Center (Amsterdam, the Netherlands), where pre-term infants ( $<37$ weeks) with a suspected diagnosis of RDS and failing nasal continuous positive airway pressure are treated with primary open lung HFOV. Infants were included in the study if HFOV was started within $72 \mathrm{~h}$ after birth and written informed consent was obtained from both parents. Exclusion criteria were congenital anomalies, severe circulatory shock or persistent pulmonary hypertension of the newborn. All patients were ventilated in the supine position and were not sedated or paralysed. The study was approved by the Institutional Review Board of the Academic Medical Centre of Amsterdam (Amsterdam, the Netherlands).

\section{Ventilation protocol}

The first phase of the study consisted of an oxygenationguided, individualised lung recruitment procedure, aiming to place ventilation on the deflation limb of the pressure-volume curve $[20,21]$. All patients were ventilated with a Sensormedics 3100A oscillator (CareFusion, Yorba Linda, CA, USA), starting at a continuous distending pressure (CDPst) of 6$8 \mathrm{cmH}_{2} \mathrm{O}$ and pressure amplitude resulting in visible oscillations of the chest, with a frequency of $10 \mathrm{~Hz}$ and an inspiration time of $33 \%$. The CDP was increased with steps of $1-2 \mathrm{cmH}_{2} \mathrm{O}$ every 2-3 min until either oxygenation no longer improved or the $\mathrm{FI}, \mathrm{O}_{2}$ was $\leqslant 0.25$ with an arterial oxygen saturation measured by pulse oximetry $\left(\mathrm{Sp}, \mathrm{O}_{2}\right)$ of $86-94 \%$ (opening pressure (CDPo)). Next, CDP was decreased with 1-2 $\mathrm{cmH}_{2} \mathrm{O}$ steps every 2-3 min until oxygenation deteriorated, indicating alveolar/saccular collapse (closing pressure $(\mathrm{CDP}))$ ). Finally, the lung was once more recruited with the known CDPo and then stabilised with a CDP $2 \mathrm{cmH}_{2} \mathrm{O}$ above CDPc (optimal pressure (CDPopt)). After confirming the correct endotracheal tube position by chest radiograph, surfactant was administered and, following a 10-min stabilisation period, the post-surfactant $\mathrm{CDP}_{\mathrm{c}}$, CDPo and CDPopt were once more determined.

$\mathrm{Sp}, \mathrm{O}_{2}$ and transcutaneous carbon dioxide pressure $\left(\mathrm{Ptc}_{\mathrm{tc}}, \mathrm{CO}_{2}\right)$ were monitored continuously throughout the recruitment procedure, but only the stabilised values that were displayed just before each pressure step were used for further analysis. Adjustments in pressure amplitude were made only if the absolute $P_{\mathrm{tc}}, \mathrm{CO}_{2}$ value was deemed to be reliable and outside the target range $(4.5-7.5 \mathrm{kPa})$ for more than three pressure steps during the recruitment procedure.
The second phase of the study started 10 min after stabilisation at the post-surfactant CDPopt. The pressure amplitude was increased by $5 \mathrm{cmH}_{2} \mathrm{O}$ for $10 \mathrm{~min}$ and then decreased back to the initial setting.

\section{EIT examination}

Before intubation, 16 hand-trimmed ECG electrodes (BlueSensor, BRS-50-K; Ambu Inc., Linthicum, MD, USA) were equidistantly placed on the thorax circumference of the newborn just above the nipple line and connected to the Goettingen Goe-MF II EIT system (CareFusion, Hoechberg, Germany). Small electrical currents (5 mArms, $100 \mathrm{kHz}$ ) were repetitively injected in rotation through adjacent electrode pairs, and voltage changes were measured by all passive electrodes pairs (scan rate $44 \mathrm{~Hz}$ ). A backprojection image reconstruction algorithm generated a $32 \times 32$ matrix of local relative impedance changes $(\Delta \mathrm{Z})$ compared with a reference state. Changes in lung electrical impedance and airway pressure were continuously recorded during the recruitment procedure and the pressure amplitude trial using the Veit software (CareFusion). EIT data were analysed off-line using AUSPEX version 1.6 (VUMC, Amsterdam, the Netherlands).

\section{Off-line analyses}

Lung recruitment and volume changes

Using the absolute pressure steps and the concomitant changes in normalised $\Delta \mathrm{Z}$, the inflation and deflation limbs of the recruitment manoeuvre were plotted for all individual patients, as previously described [22]. Next, the inflation limb was fitted according to the model described by VENEGAS et al. [14], which is formulated as:

$$
V=\mathrm{a}+\mathrm{b} /\left(1+\mathrm{e}^{-(P-\mathrm{c}) / \mathrm{d}}\right)
$$

where $V$ is lung volume, $P$ is the pressure at the airway opening, $\mathrm{a}$ is the lower asymptote volume, $\mathrm{b}$ is the total change in lung volume between the lower and upper asymptote, c corresponds to the pressure at the point of highest compliance and $\mathrm{d}$ is proportional to the pressure range within which most of the volume change takes place. Using this formula, we determined the pressure at which maximal respiratory system compliance (Crs,max ) was reached [14].

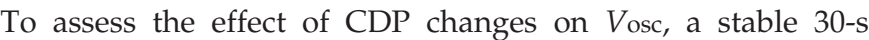
period was selected at each pressure step during the recruitment procedure and referenced to the average $\Delta \mathrm{Z}$ in that same period. Next, the $\Delta Z$ signal was high-pass filtered leaving only $\Delta Z$ changes that occurred at a frequency of $>580$ per min $(10 \mathrm{~Hz})$. The oscillation amplitudes were calculated using the peaks and troughs of this signal. The averaged oscillation amplitudes of $\Delta \mathrm{Z}$ values and the concomitant changes in $P \mathrm{tc}, \mathrm{CO}_{2}$ levels were then plotted against the absolute pressure changes for both the inflation and deflation limbs. The derivative of the Venegas equation was used to calculate the pressure corresponding with the maximal $V_{\text {osc }}$ and the lowest $P_{\mathrm{tc},} \mathrm{CO}_{2}$ level of the inflation limb [14].

The analyses described above were also performed for the $V_{\text {osc }}$ in the ventral, dorsal, right and left halves of the cross-section.

Pressure amplitude and volume changes

To establish the effect of pressure amplitude changes on $V_{\text {osc }}$, 30 -s periods were selected at the end of each 10-min period 
before, during and after the increase in pressure amplitude and referenced to the average $\Delta Z$ in that same period. For each of these three periods, the EIT signal was band-pass filtered in the spontaneous breathing domain (5-90 per min) and the spontaneous tidal breathing frequency was determined. In addition, the signal was high-pass filtered ( $>580$ per min), focussing only on the oscillation frequency and its higher harmonics to determine the change in $V_{\text {osc, }}$ expressed as a percentage of the starting value.

Using the same 30-s periods, we also assessed the effect of changed pressure amplitude on lung volume, by using a fixed reference period selected at the start of the intervention.

\section{Statistical analysis}

For statistical analysis, we used GraphPad Prism 5.0 (GraphPad Software Inc., San Diego, CA, USA) and SPSS version 16.0 (SPSS Inc., Chicago, IL, USA). Depending on their distribution, data were expressed as mean \pm SD or as median with interquartile range (IQR). For comparative analyses, a MannWhitney or Wilcoxon rank test were used for skewed data and a paired t-test for normal distributed data. Bivariate nonparametric correlations (Spearman's rank correlation coefficient, $\rho)$ were calculated for $V_{\text {osc }}$ versus $P_{\mathrm{tc}}, \mathrm{CO}_{2}$ and between pressure at $\mathrm{Crs}$,max, maximal $V_{\mathrm{osc}}$ and minimal $\mathrm{Ptc}_{\mathrm{tc}} \mathrm{CO}_{2}$. A p-value of $<0.05$ was considered statistically significant.

\section{RESULTS}

10 newborn infants were included in the study and completed the recruitment procedure and the change in pressure amplitude without complications (table 1). The mean \pm SD CDPst was $7.6 \pm 1.3 \mathrm{cmH}_{2} \mathrm{O}$ with a $\mathrm{FI}_{1} \mathrm{O}_{2}$ of $0.73 \pm 0.24$. The recruitment procedure resulted in $\mathrm{CDP}_{\mathrm{o}} \mathrm{CDP}_{\mathrm{c}}$ and CDPopt of $19.2 \pm 2.1$, $10.1 \pm 1.5$ and $12.1 \pm 1.5 \mathrm{~cm} \mathrm{H}_{2} \mathrm{O}$, respectively, with a reduction in $\mathrm{F}, \mathrm{O}_{2}$ to $0.24 \pm 0.03$. The pressure amplitude at the start of recruitment was $20.6 \pm 2.3 \mathrm{~cm} \mathrm{H}_{2} \mathrm{O}$ and it was maintained during the pre-surfactant recruitment procedure. The change in pressure amplitude in the second phase of the study after surfactant administration was performed at a CDPopt of

\begin{tabular}{lccccc} 
TABLE 1 & \multicolumn{6}{c}{ Patient characteristics } & & \\
$\begin{array}{l}\text { Subject } \\
\text { no. }\end{array}$ & $\begin{array}{c}\text { GA } \\
\text { weeks }\end{array}$ & $\begin{array}{c}\text { Weight } \\
\mathbf{g}\end{array}$ & $\begin{array}{c}\mathbf{5}^{\prime} \text { ' } \mathbf{\text { Apgar }} \\
\text { score }\end{array}$ & $\begin{array}{c}\text { Age at } \\
\text { intubation } \mathbf{~}\end{array}$ & $\mathbf{m O I}$ \\
\hline $\mathbf{1}$ & 25.3 & 750 & 6 & 3 & 4.5 \\
$\mathbf{2}$ & 28.1 & 760 & 9 & 4 & 4.5 \\
$\mathbf{3}$ & 25.6 & 790 & 7 & 1 & 12.0 \\
$\mathbf{4}$ & 29.3 & 1095 & 8 & 2 & 3.1 \\
$\mathbf{5}$ & 28.7 & 880 & 8 & 24 & 6.7 \\
$\mathbf{6}$ & 29.6 & 1000 & 4 & 10 & 5.3 \\
$\mathbf{7}$ & 26.3 & 1020 & 9 & 2 & 11.4 \\
$\mathbf{8}$ & 28.0 & 840 & 9 & 3 & 4.3 \\
$\mathbf{9}$ & 27.0 & 610 & 8 & 3 & 8.8 \\
$\mathbf{1 0}$ & 28.6 & 775 & 9 & 4 & 4.7 \\
Median & 27.1 & 815 & 8 & 3 & 5.0 \\
\hline
\end{tabular}

GA: gestational age; mOl: modified oxygenation index (continuous distending pressure $\times$ fraction of inspired oxygen (\%)/oxygen saturation).
$7.2 \pm 1.0 \mathrm{cmH}_{2} \mathrm{O}$ and resulted in an increase from $18.2 \pm 2.3$ $\mathrm{cmH}_{2} \mathrm{O}$ to $23.2 \pm 2.3 \mathrm{cmH}_{2} \mathrm{O}$.

\section{Lung recruitment and volume changes}

All individual pressure-impedance curves showed clear lung hysteresis (fig. 1). Modelling of the inflation limb according to the Venegas equation was possible in all patients with a goodness of fit $\left(R^{2}\right)$ of $0.99 \pm 0.00$ resulting in a median (IQR) pressure at $\mathrm{Crs}$,max of $12.9(12.1-16.7) \mathrm{cmH}_{2} \mathrm{O}$. The changes in $V$ osc and $\mathrm{Ptc}_{\mathrm{tc}} \mathrm{CO}_{2}$ showed a significant, inverse correlation in all patients during the recruitment procedure (figs S1A and S1B). As expected, the pressure $-V_{\mathrm{osc}}$ and pressure $-P_{\mathrm{tc},} \mathrm{CO}_{2}$ relationships during inflation showed a parabolic shape in almost all infants (fig. 1). The derivative of the Venegas equation could be fitted for the $V_{\text {osc }}$ in 10 patients $\left(R^{2}=0.92 \pm 0.06\right)$ and for the $P \mathrm{tc}, \mathrm{CO}_{2}$ data in eight patients $\left(\mathrm{R}^{2}=0.95 \pm 0.03\right)$. The pressure at maximal $V_{\text {osc }}$ determined from these fitted curves during inflation was $14.0(13.0-14.5) \mathrm{cmH}_{2} \mathrm{O}$. For the minimal $P_{\mathrm{tc},} \mathrm{CO}_{2}$, it was $13.7(11.8-14.8) \mathrm{cmH}_{2} \mathrm{O}$. Both pressures were significantly correlated with $\mathrm{Crs}$,max pressure (fig. 2). During the decremental pressure steps, the pressure $-V_{\text {osc }}$ and pressure $-P_{\mathrm{tc}}, \mathrm{CO}_{2}$ relationships could not be fitted due to insufficient number of data points, but in all patients, CDP reduction from CDPo resulted in an (initial) increase in $V_{\text {osc }}$ (figs 1, S1A and S1B).

Regional analysis of the data from the ventral versus dorsal and right versus left cross-section halves of the Vosc showed similar results in pressure at $C_{r s, \max }$ maximal $V_{\text {osc }}$ and minimal

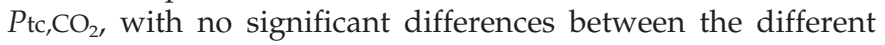
regions of interest.

\section{Pressure amplitude and volume changes}

Increasing the pressure amplitude by $5 \mathrm{cmH}_{2} \mathrm{O}$ resulted in a significant increase in the median $V_{\text {osc }}$ and a concomitant decrease in $\mathrm{Ptc}_{\mathrm{tc}} \mathrm{CO}_{2}$ (fig. 3). This increase in $V_{\mathrm{osc}}$ was evenly distributed over the ventral and dorsal lung regions. Comparing the right and left lungs revealed that the volume increase was significantly higher in the right lung (data not shown). The increase in pressure amplitude resulted in a significant $(\mathrm{p}<0.01)$ decrease in the spontaneous breathing frequency from $36(20-76)$ to $18(10-30)$ per min. Functional residual capacity showed no significant change in response to the increase in pressure amplitude.

\section{DISCUSSION}

HFOV is a lung protective ventilation mode that is used in 15$25 \%$ of pre-term infants $[1,23]$. It has been suggested that oxygenation and ventilation during HFOV can be controlled independently by adjusting, respectively, lung volume via the CDP and the Vosc via the pressure amplitude [3-6]. This study shows, for the first time, that this assumption is not correct for pre-term infants with RDS during lung volume recruitment following the initiation of HFOV.

The most important finding of this study is that pulmonary ventilation and gas exchange, expressed as both $V_{\text {osc }}$ and $P \mathrm{tc}, \mathrm{CO}_{2}$ levels, changes during a lung recruitment procedure on $\mathrm{HFOV}$, despite the fact that the pressure amplitude remains constant. The changes in $V_{\mathrm{osc}}$ and $\mathrm{Ptc}_{\mathrm{tc}} \mathrm{CO}_{2}$ were inversely correlated in all patients, strengthening the validity of our findings. The pattern of these changes during inflation was similar in most of the patients, showing an increase in Vosc and a decrease 


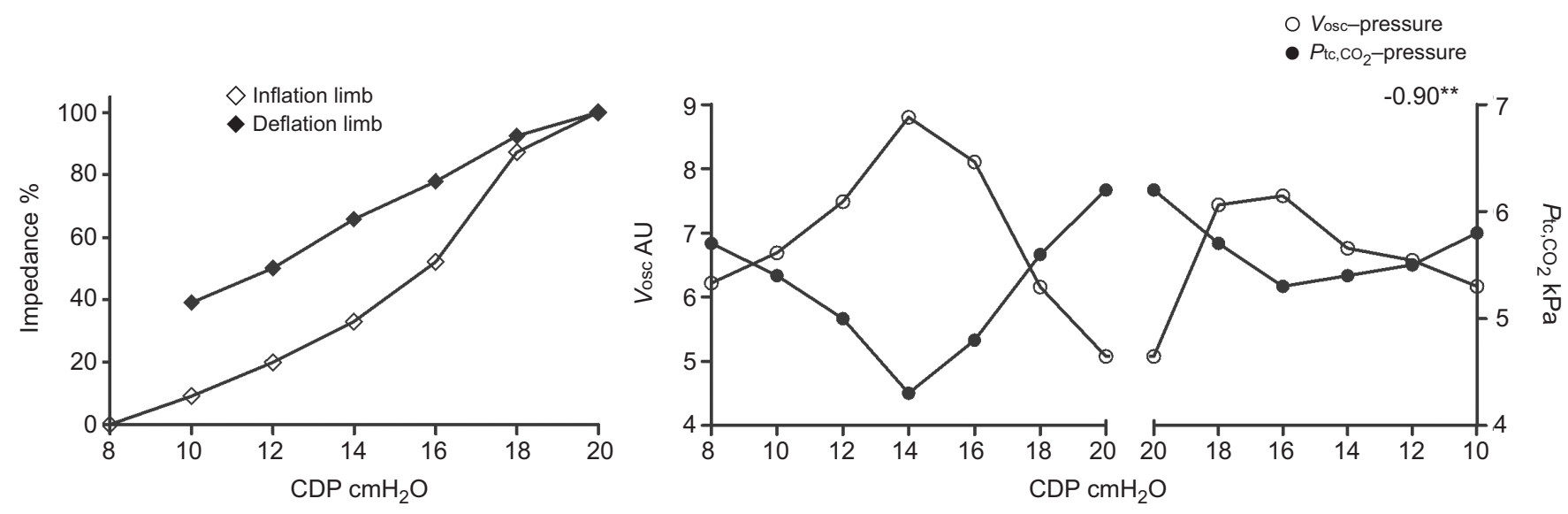

FIGURE 1. Representative global pressure-impedance (left panel), oscillation volume (Vosc)-pressure and transcutaneous carbon dioxide pressure $\left(P_{t c}, \mathrm{CO}_{2}\right)$-pressure relationships during the inflation (middle panel) and deflation limb (right panel) of an open lung high-frequency ventilated pre-term infant. The $X$-axis of the deflation limb is shown in the reverse order from high to low continuous distending pressures (CDP). The Spearman's rank correlation coefficients between $V_{o s c}$ and $P_{t c}, \mathrm{CO}_{2}$ is given in the right upper corner. ${ }^{*}: p<0.01$.

in $P_{\mathrm{tc}, \mathrm{CO}_{2}}$ during the first part of recruitment, followed by a decrease in $V$ osc and an increase in $P_{\mathrm{tc},}, \mathrm{CO}_{2}$ towards the end of the incremental pressure steps. The fact that the airway pressures resulting in the maximal compliance, the highest $V_{\text {osc }}$ and the lowest $\mathrm{Ptc}_{\mathrm{tc}} \mathrm{CO}_{2}$ were almost identical and highly correlated strongly suggests that the observed ventilation pattern is best explained by the changes in lung compliance as ventilation moves up the inflation limb of the pressure-volume curve. Despite only few data points being available during deflation, the increase in $V_{\mathrm{osc}}$ with a concomitant decrease in $\mathrm{Ptc}_{\mathrm{tc}} \mathrm{CO}_{2}$ seems to support the association between lung volume and ventilation. Our findings are in line with the study of VAN GENDERINGEN $e t$ al. [24], showing a similar relationship between FRC and ventilation, expressed as the oscillatory pressure ratio, in highfrequency ventilated surfactant-deficient pigs. The fact that the

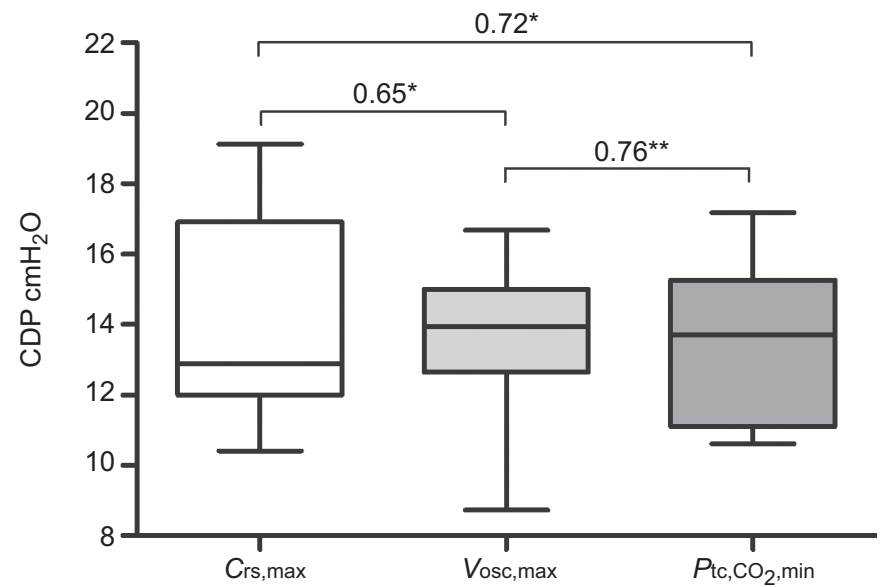

FIGURE 2. Continuous distending pressures (CDPs) corresponding with maximal respiratory system compliance (Crs,max), maximal oscillation volume (Vosc,max) and minimal transcutaneous pressure of carbon dioxide $\left(\mathrm{Ptc}_{\mathrm{tc}} \mathrm{CO}_{2}, \mathrm{~min}\right)$ during lung inflation. The Spearman's rank correlation coefficients between these three variables are shown above the box plots. The median, 25th and 75th percentiles, and the minimum and maximum values of 10 infants are shown. $*: p<0.05 ; * *: p<0.01$ association between FRC and (tidal) ventilation was also found in animal experiments using pressure-controlled ventilation indicates that these physiological principles are independent of the ventilation mode $[15,18]$. In an in vitro study, PILlow et al. [25] also found a clear association between changes in lung compliance and tidal volumes during HFOV. However, this association disappeared when compliance exceeded a certain threshold, indicating that the findings of our study may have specific relevance to recruitment from atelactasis and are less important when lung volume is maintained at optimal CDP.

An important advantage of EIT is its ability to assess the regional changes in lung aeration. Our study shows that the changes in $V$ osc during lung recruitment are evenly distributed

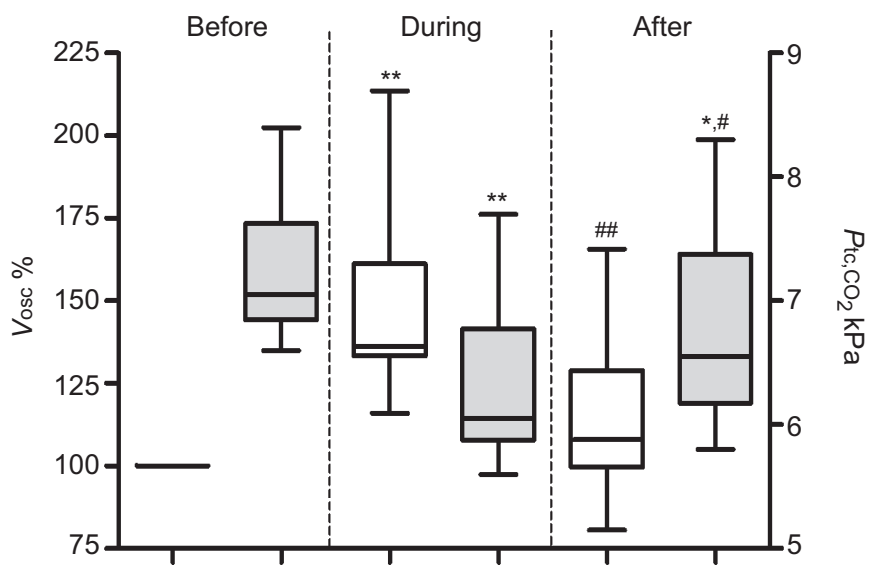

FIGURE 3. Oscillation volumes (Vosc; white box) and transcutaneous carbon dioxide pressure $\left(\mathrm{Ptc}_{\mathrm{tc}} \mathrm{CO}_{2}\right.$; grey box) levels before, during and after a $5-\mathrm{cmH}_{2} \mathrm{O}$ increase in pressure amplitude with high-frequency oscillatory ventilation in preterm infants. The initial Vosc before the pressure amplitude increase was set at $100 \%$. The median, 25 th and 75 th percentile, and the minimum and maximum values of 10 infants are shown. ${ }^{*}: p<0.05$ after versus before the increase in pressure amplitude; ${ }^{*}: p<0.01$ during versus before the increase in pressure amplitude; ${ }^{\#:} p<0.05$ after versus during the increase in pressure amplitude; \#\#: $p<0.01$ after versus during the increase in pressure amplitude. 
across the ventral and dorsal lung regions, supporting previous findings that RDS is a relatively homogenous lung disease [21, 22].

Previous studies have shown that an increase in pressure amplitude increases the $V_{\text {osc }}$ measured at the airway opening [6-8]. This study shows that this increase in Vosc can also be detected at the regional pulmonary level using EIT. Furthermore, we were able to show that this increase in $V_{\text {osc }}$ also resulted in a decrease in $P \mathrm{tc}, \mathrm{CO}_{2}$ and the spontaneous breathing frequency, strengthening the validity of our EIT finding. Again, the increase in $V$ osc was evenly distributed across the ventral and dorsal lung regions. We did, however, find a significantly higher increase in $V_{\text {osc }}$ in the right lung compared with the left lung. This right-sided predominance is probably best explained by the presence of the heart in the left hemithorax $[21,26]$.

The increase in pressure amplitude did not seem to impact on FRC, but this finding needs to be interpreted with some caution because the variation in pressure amplitude was relatively modest and only applied at one point in time during HFOV when the lung was already at optimal CDP. Furthermore, the pressure amplitude change was confined to a short interval. The results may be different if the change in pressure amplitude was more substantial or applied at different stages of lung recruitment, lung disease and at different ventilator settings. To our knowledge, only one study varied the pressure amplitude repeatedly in pre-term infants on HFOV, but unfortunately this study did not measure FRC [9]. However, the authors did report a modest but significant increase in arterial oxygen pressure $(1.1 \mathrm{kPa})$, which may have reflected an increase in lung volume.

This study has several limitations that need to be addressed. First, EIT only provides information on a transverse "slice" of the lung. Considering the fact that RDS is a relatively homogeneous lung disease, it is very likely that the EIT findings as described in this study are representative of the entire lung. The close association between the cross-sectional changes in $V_{\text {osc }}$ and $\mathrm{Ptc}_{\mathrm{tc}} \mathrm{CO}_{2}$ seem to support this assumption. Secondly, this study only included pre-term infants with RDS and the findings may be different in other causes of respiratory failure. Finally, although not essential, this study did not provide information on the absolute changes in $V$ osc. Unfortunately, calibration of the electrical impedance tomography signal to tidal volumes measured at the airway opening is not yet feasible.

Despite these limitations and the fact that EIT remains a research tool, our study has important implications for clinicians using HFOV in pre-term infants. First, this study shows that, in addition to oxygenation, changes in $V$ osc and $\mathrm{Ptc}_{\mathrm{tc}}, \mathrm{CO}_{2}$ can also assist the clinician in optimising the lung volume during HFOV. Transcutaneous monitoring is nowadays increasingly used in clinical practice and many of the newer ventilators display (a measure of) tidal volumes during HFOV. During the incremental pressure steps in particular, falling $V_{\text {osc }}$ or an increase in pressure amplitude in case of volume guarantee and an increase in $P \mathrm{tc}, \mathrm{CO}_{2}$, can alert the clinician that he or she is approaching the flat part of the inflation limb, i.e. optimal recruitment. Secondly, clinicians should closely monitor $\mathrm{Ptc}_{\mathrm{tc}} \mathrm{CO}_{2}$ during lung recruitment and if necessary, adjust the pressure amplitude in order to minimise the risk of hypo- and hypercapnia. Finally, in those infants that are not heavily sedated during HFOV, changes in spontaneous breathing activity seem to be associated with oscillatory volume and $\mathrm{Ptc}_{\mathrm{tc}} \mathrm{CO}_{2}$. This finding suggests that spontaneous breathing activity can be used in a clinical strategy that aims to preserve normal breathing during HFOV, thereby reducing the risk of overventilation and diaphragmatic dysfunction.

In conclusion, this study shows that the oscillatory volumes during HFOV in pre-term infants with RDS is closely related to the position of ventilation on the inflation and deflation limb of the pressure-volume relationship of the lung, and the applied pressure amplitude. Changes in pressure amplitude do not seem to impact the FRC. This information can help the clinician in optimising lung volume and ventilation during HFOV.

\section{STATEMENT OF INTEREST}

A statement of interest for this study can be found at www.erj. ersjournals.com/site/misc/statements.xhtml

\section{ACKNOWLEDGEMENTS}

The EIT device was kindly provided by CareFusion.

\section{REFERENCES}

1 van Kaam AH, Rimensberger PC, Borensztajn D, et al. Ventilation practices in the neonatal intensive care unit: a cross-sectional study. J Pediatr 2010; 157: 767-771.

2 Dimitriou G, Greenough A. Measurement of lung volume and optimal oxygenation during high frequency oscillation. Arch Dis Child Fetal Neonatal Ed 1995; 72: F180-F183.

3 Goldstein D, Slutsky AS, Ingram $\mathrm{RH} \mathrm{Jr}$, et al. $\mathrm{CO}_{2}$ elimination by high frequency ventilation $(4$ to $10 \mathrm{~Hz}$ ) in normal subjects. Am Rev Respir Dis 1981; 123: 251-255.

4 Lampland AL, Mammel MC. High Frequency Ventilation. In: Goldsmith JP, Karotkin EH, eds. Assisted Ventilation of the Neonate. St Louis, Elsevier Saunders, 2011; pp. 200-219.

5 Simon BA, Weinmann GG, Mitzner W. Mean airway pressure and alveolar pressure during high-frequency ventilation. J Appl Physiol 1984; 57: 1069-1078.

6 Slutsky AS, Kamm RD, Rossing TH, et al. Effects of frequency, tidal volume, and lung volume on $\mathrm{CO}_{2}$ elimination in dogs by high frequency $(2-30 \mathrm{~Hz})$, low tidal volume ventilation. J Clin Invest 1981; 68: 1475-1484.

7 Boynton BR, Hammond MD, Fredberg JJ, et al. Gas exchange in healthy rabbits during high-frequency oscillatory ventilation. J Appl Physiol 1989; 66: 1343-1351.

8 Kamitsuka MD, Boynton BR, Villanueva D, et al. Frequency, tidal volume, and mean airway pressure combinations that provide adequate gas exchange and low alveolar pressure during high frequency oscillatory ventilation in rabbits. Pediatr Res 1990; 27: 64-69.

9 Morgan C, Dear PR, Newell SJ. Effect of changes in oscillatory amplitude on $\mathrm{PaCO}(2)$ and $\mathrm{PaO}(2)$ during high frequency oscillatory ventilation. Arch Dis Child Fetal Neonatal Ed 2000; 82: F237-F242.

10 van Genderingen HR, van Vught JA, Jansen JR, et al. Oxygenation index, an indicator of optimal distending pressure during highfrequency oscillatory ventilation? Intensive Care Med 2002; 28: 1151-1156.

11 Boynton BR, Villanueva D, Hammond MD, et al. Effect of mean airway pressure on gas exchange during high-frequency oscillatory ventilation. J Appl Physiol 1991; 70: 701-707.

12 Hickling KG. The pressure-volume curve is greatly modified by recruitment. A mathematical model of ARDS lungs. Am J Respir Crit Care Med 1998; 158: 194-202. 
13 Hickling KG. Best compliance during a decremental, but not incremental, positive end-expiratory pressure trial is related to open-lung positive end-expiratory pressure: a mathematical model of acute respiratory distress syndrome lungs. Am J Respir Crit Care Med 2001; 163: 69-78.

14 Venegas JG, Harris RS, Simon BA. A comprehensive equation for the pulmonary pressure-volume curve. J Appl Physiol 1998; 84: 389-395.

15 Dargaville PA, Rimensberger PC, Frerichs I. Regional tidal ventilation and compliance during a stepwise vital capacity manoeuvre. Intensive Care Med 2010; 36: 1953-1961.

16 Victorino JA, Borges JB, Okamoto VN, et al. Imbalances in regional lung ventilation: a validation study on electrical impedance tomography. Am J Respir Crit Care Med 2004; 169: 791-800.

17 Bikker IG, Leonhardt S, Reis MD, et al. Bedside measurement of changes in lung impedance to monitor alveolar ventilation in dependent and non-dependent parts by electrical impedance tomography during a positive end-expiratory pressure trial in mechanically ventilated intensive care unit patients. Crit Care 2010; 14: R100.

18 Meier T, Luepschen H, Karsten J, et al. Assessment of regional lung recruitment and derecruitment during a PEEP trial based on electrical impedance tomography. Intensive Care Med 2008; 34: 543-550.

19 Hinz J, Moerer O, Neumann P, et al. Regional pulmonary pressure volume curves in mechanically ventilated patients with acute respiratory failure measured by electrical impedance tomography. Acta Anaesthesiol Scand 2006; 50: 331-339.

20 De Jaegere A, van Veenendaal MB, Michiels A, et al. Lung recruitment using oxygenation during open lung high-frequency ventilation in preterm infants. Am J Respir Crit Care Med 2006; 174: 639-645.

21 Miedema M, de Jongh FH, Frerichs I, et al. Changes in lung volume and ventilation during lung recruitment in high-frequency ventilated preterm infants with respiratory distress syndrome. J Pediatr 2011; 159: 199-205.

22 Miedema M, de Jongh FH, Frerichs I, et al. Changes in lung volume and ventilation during surfactant treatment in ventilated preterm infants. Am J Respir Crit Care Med 2011; 184: 100-105.

23 Horbar JD, Badger GJ, Carpenter JH, et al. Trends in mortality and morbidity for very low birth weight infants, 1991-1999. Pediatrics 2002; 110: 143-151.

24 van Genderingen HR, van Vught AJ, Duval EL, et al. Attenuation of pressure swings along the endotracheal tube is indicative of optimal distending pressure during high-frequency oscillatory ventilation in a model of acute lung injury. Pediatr Pulmonol 2002; 33: 429-436.

25 Pillow JJ, Wilkinson $\mathrm{MH}$, Neil $\mathrm{HL}$, et al. In vitro performance characteristics of high-frequency oscillatory ventilators. Am J Respir Crit Care Med 2001; 164: 1019-1024.

26 Frerichs I, Schiffmann H, Hahn G, et al. Non-invasive radiationfree monitoring of regional lung ventilation in critically ill infants. Intensive Care Med 2001; 27: 1385-1394. 